\title{
Voltage Gating of a Biomimetic Nanopore: Electrowetting of a Hydrophobic Barrier
}

\author{
Jemma L. Trick, ${ }^{\dagger, \S}$ Chen Song, ${ }^{\dagger, \perp}$ E. Jayne Wallace, ${ }^{\dagger}$ and Mark S. P. Sansom* ${ }^{\dagger \dagger}$ \\ ${ }^{\dagger}$ Department of Biochemistry, University of Oxford, Oxford OX1 3QU, U.K. \\ ${ }^{\ddagger}$ Oxford Nanopore Technologies Ltd., Edmund Cartwright House, 4 Robert Robinson Avenue, Oxford Science Park, Oxford OX4 \\ 4GA, U.K.
}

\section{Supporting Information}

\begin{abstract}
It is desirable that nanopores that are components of biosensors are gated, i.e., capable of controllable switching between closed (impermeable) and open (permeable) states. A central hydrophobic barrier within a nanopore may act as a voltage-dependent gate via electrowetting, i.e., changes in nanopore surface wettability by application of an electric field. We use "computational electrophysiology" simulations to demonstrate and characterize electrowetting of a biomimetic nanopore containing a hydrophobic gate. We show that a hydrophobic gate in a model $\beta$-barrel nanopore can be functionally opened by electrowetting at voltages that do not electroporate lipid bilayers. During the process of electrowetting, voltage-induced alignment of water dipoles occurs within the hydrophobic gate region of the nanopore, with water entry preceding permeation of ions through the opened nanopore. When the ionic imbalance that generates a transbilayer potential is dissipated,

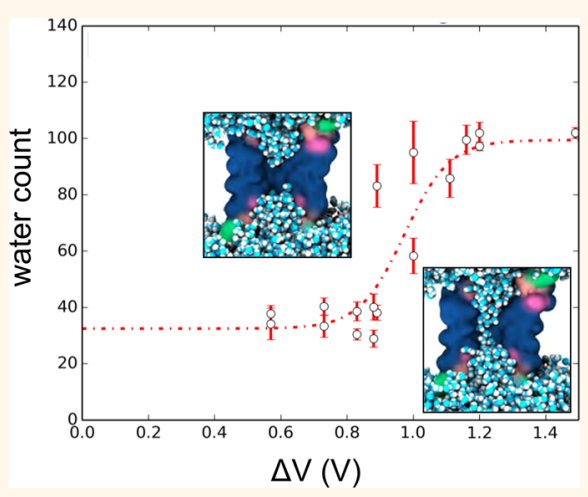
water is expelled from the hydrophobic gate and the nanopore recloses. The open nanopore formed by electrowetting of a "featureless" $\beta$-barrel is anionic selective due to the transmembrane dipole potential resulting from binding of $\mathrm{Na}^{+}$ions to the headgroup regions of the surrounding lipid bilayer. Thus, hydrophobic barriers can provide voltage-dependent gates in designed biomimetic nanopores. This extends our understanding of hydrophobic gating in synthetic and biological nanopores, providing a framework for the design of functional nanopores with tailored gating functionality.
\end{abstract}

KEYWORDS: nanopore, membrane, hydrophobic barrier, electrowetting, molecular dynamics simulation

$\mathrm{N}$ anopores in membranes ${ }^{1}$ have considerable potential as biosensors, ${ }^{2,3}$ in applications ranging from water desalination ${ }^{4}$ to DNA sequencing. ${ }^{5,6}$ Nanopores may be formed from a range of materials, both biological (proteins, peptides, ${ }^{8,9} \mathrm{DNA}^{10,11}$ ) and nonbiological (e.g., carbon nanotubes $^{12}$ and track etched nanopores ${ }^{13}$ ). For such systems to be fully exploited, nanopores need to be gated, i.e., capable of controllable switching between functionally closed (impermeable) and open (permeable) states.

A promising design of a potential gate in a nanopore is the presence of a hydrophobic barrier within the pore. ${ }^{14}$ Such a region may be sterically unoccluded but of sufficient local hydrophobicity to "dewet", i.e., to exclude water molecules and hence prevent permeation of both water molecules and ions. ${ }^{15}$ Such a hydrophobic region in a nanopore has been the subject of some interest and has been variously referred to as a hydrophobic gate, ${ }^{16}$ barrier, ${ }^{17}$ vapor lock, ${ }^{18,19}$ or simply a hydrophobic region leading to local dewetting of a pore. The properties of such hydrophobic barriers in nanopores and their dependence on local pore geometry, hydrophobicity, and flexibility have formed the subject of numerous computational studies of hydrophobic gates in simple models, ${ }^{14,15,20-25}$ in nanopores formed by carbon nanotubes, ${ }^{26-28}$ and in biological pores. ${ }^{16,29-31}$ However, rather less attention has been paid to how such gates may be switched between closed and open states in a controlled fashion, even though this is of central importance in the design of functional nanopores for a number of applications.

Electrowetting, whereby surface wettability is controlled by application of an electric field, ${ }^{32}$ is an attractive possibility for controlled opening of a gate formed by a hydrophobic region of a nanopore. ${ }^{23}$ Electrowetting has been demonstrated in simulation studies of simple model nanopores ${ }^{33,34}$ and of carbon nanotubes. ${ }^{27,35}$ It has also been observed experimentally in, for example, nanopores in polymer $^{13}$ or silicon nitride membranes that have chemically derivatized hydrophobic linings. ${ }^{36}$ Simulation studies have also suggested that electrowetting may occur in some protein channels, ${ }^{37}$ e.g., $\mathrm{MscS}^{29}$ and TWIK-1 ${ }^{17}$ channels.

Received: November 22, 2016

Accepted: January 31, 2017

Published: January 31, 2017 
From these studies it has been concluded that electrowetting of nanopores may be attributed to the orientation of the water dipoles in response to an electric field, leading to a change in water density in the hydrophobic regions of the nanopores and hence a wetting event. ${ }^{23,25,36}$ It has been suggested that this is thermodynamically favorable for a range of nanopore diameters but may be kinetically realistic only in small-diameter nanopores. ${ }^{36}$ Simulations of pores formed by carbon nanotubes $^{27}$ revealed that a two-state model of water dipole orientation in the nanotube describes electrowetting of the pore. Simple models of hydrophobic nanopores between two reservoirs containing an unequal number of cations have been shown to undergo electrowetting, with the nanopore-confined water undergoing strong electroconstriction. ${ }^{23}$ The electric field strength that initiates water permeation was sensitive to the length and radius of the pore. For a model nanopore-like region between two disks of butylated graphene, application of an electric field enhanced uptake of both water and ions into the confined region. ${ }^{38}$

Experimentally, transmembrane electric fields may be generated by an ionic imbalance across a lipid bilayer. In simulations this may be modeled either via imposing a constant electric field along the bilayer normal ${ }^{39}$ or by explicitly simulating the imbalance in ion concentrations across the membrane. ${ }^{40}$ The relationship between the two approaches has been explored, suggesting that they are similar in their effects on structural properties of a bilayer membrane. ${ }^{41}$ The ionic imbalance approach, which has been referred to as "computational electrophysiology" (CE) ${ }^{42}$ permits one to simulate directly electrowetting of nanopores induced by a gradient in ionic concentrations, thus closely mimicking experiments. Here we use $\mathrm{CE}$ to demonstrate and characterize electrowetting of a biomimetic nanopore containing a hydrophobic gate, showing that this permits controllable and reversible voltage gating of a nanopore in a lipid bilayer membrane.

\section{RESULTS AND DISCUSSION}

Model Protein Nanopores with a Hydrophobic Barrier. Our model protein nanopores were derived from those designed in a previous study, ${ }^{43}$ consisting of a membrane spanning a $\beta$-barrel with a central hydrophobic barrier formed by rings of leucine side chains. On the basis of previous simulations of wetting/dewetting within a nanopore due to a central hydrophobic barrier, ${ }^{43}$ we selected two $\beta$-barrels formed by either $14 \beta$-strands with three rings of hydrophobic (leucine; $\mathrm{L})$ side chains $(\beta 14 \mathrm{~L} 3$; Figure $1 \mathrm{~A})$ or $16 \beta$-strands with five rings of hydrophobic side chains $(\beta 16 \mathrm{~L} 5)$. Both of these $\beta$ barrels form membrane-spanning nanopores, with a minimum central pore radius of $c a .5 .5$ and $6.5 \AA$ for $\beta 14 \mathrm{~L} 3$ and $\beta 16 \mathrm{~L} 5$, respectively. In each case simulations in the absence of a transbilayer potential indicate that the pore is dewetted in the vicinity of the central hydrophobic ring, thus rendering the pore impermeable (i.e., "closed") to either water or ions. Therefore, both pores are suitable for investigating whether voltageinduced wetting would result in "opening" of a central hydrophobic gate.

Electrowetting of the $\beta 14 \mathrm{~L} 3$ Nanopore. Two methods were initially explored to simulate potential electrowetting of the $\beta 14 \mathrm{~L} 3$ nanopore: application of a constant electric field across the simulation box ${ }^{39}$ and computational electrophysiology. ${ }^{42}$ The two methods have been demonstrated to be similar in terms of the fall in electrostatic potential across the lipid bilayer. ${ }^{41}$ However, the $\mathrm{CE}$ method provides a more direct
A $\beta 14 L 3$

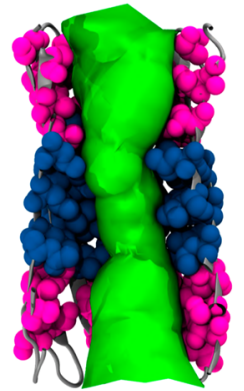

B16L5

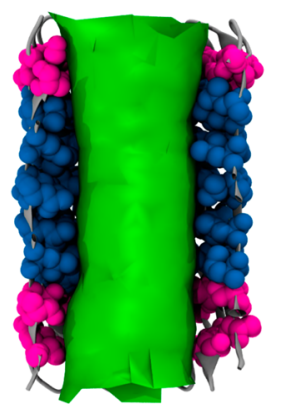

B

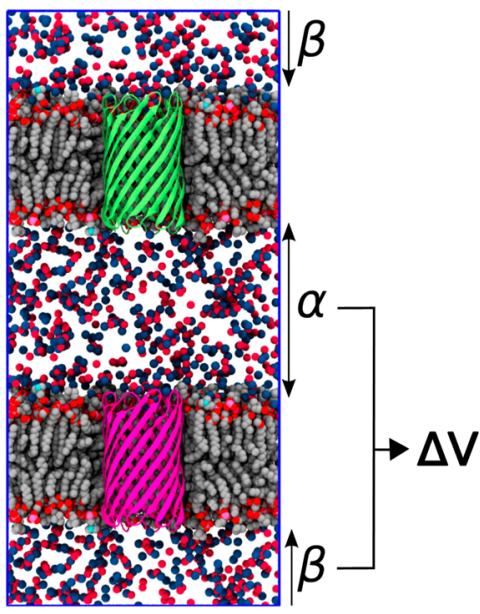

Figure 1. (A) Model nanopores with a central hydrophobic barrier to ion and water permeation. Both are transmembrane $\beta$-barrels: $\beta 14 \mathrm{~L} 3$ has a barrel formed by $14 \beta$-strands with three rings (in blue) of leucine residues forming the hydrophobic barrier; $\beta 16 \mathrm{LS}$ has $16 \beta$-strands and five rings of leucine residues forming the barrier. In each case the pore is shown "cut open" with the protein backbone in gray and the lumen-facing residues shown in spacefilling representation (pink $=$ hydrophilic side chains, blue $=$ leucine side chains). The pore lining surface (calculated using $\mathrm{HOLE}^{68}$ ) is shown in green. (B) Setup of double-bilayer system used in the computational electrophysiology simulations. Each bilayer shows the lipids (gray/red) and the inserted nanopore (green or pink). The two compartments $\alpha$ and $\beta$ have differing ion concentrations $\left(\right.$ red $=\mathrm{Na}^{+}$; blue $\left.=\mathrm{Cl}^{-}\right)$to create a transmembrane voltage difference $\Delta V=V(\alpha)-V(\beta)$ (see text and SI Figure S1 for further details). Water molecules are omitted for clarity.

simulation of experimental measurements on nanopores in that the transbilayer electric field is maintained by an asymmetric distribution of anions and cations (Figure 1B). ${ }^{42}$ Consequently, it is more straightforward to define the transmembrane potential difference for the CE method (see SI Figure S1). We will therefore focus largely on simulations using the $\mathrm{CE}$ method.

Both methods revealed two states of the pore: a "closed" state with a dewetted central region was observed in the absence of and at low transbilayer voltages (e.g., $\Delta V=0.3 \mathrm{~V}$ ), whereas an "open" state with a continuous water-filled pore was observed at higher $(\Delta V>1 \mathrm{~V})$ voltages (Figure 2 and SI Figure S2A).

As mentioned above, in the $\mathrm{CE}$ an ionic imbalance is imposed and maintained (see Methods) to create a transbilayer voltage across each of the two membranes in the simulation system (Figure 1B). Thus, in these initial simulations an ion swap of 18 cations (yielding $1187 \mathrm{Na}^{+}$ions in the $\alpha$ region and $1205 \mathrm{Na}^{+}$ions in the $\beta$ region with 1196 chloride ions in each region; Figure 1B) generated a transbilayer voltage difference of $\Delta V=1.2 \mathrm{~V}$ across each bilayer (see Methods and SI Figure $\mathrm{S} 1 \mathrm{AB}$ for details of the measurement of the transbilayer $\Delta V$ ) 

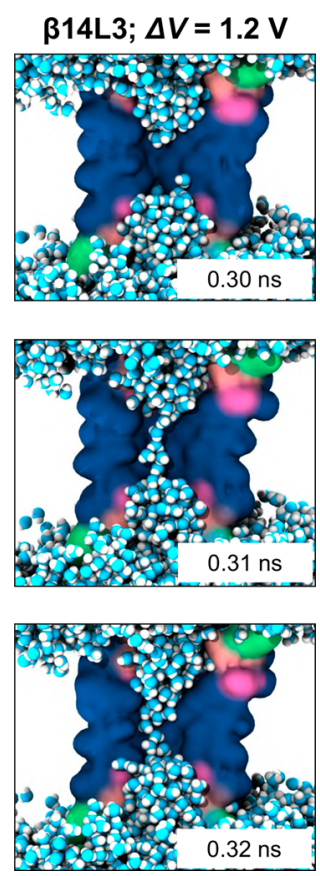

Figure 2. Water entry into the $\beta 14 \mathrm{~L} 3$ pore at $\Delta V=1.2 \mathrm{~V}$. The water molecules are shown as cyan (oxygen) and white (hydrogen) spheres; the protein is shown as a molecular surface (sliced open to show the interior of the pore). Ions and lipid molecules are omitted for clarity. The pore is shown at time $0.30 \mathrm{~ns}$ (dewetted), $0.31 \mathrm{~ns}$ (water molecules having entered to form a "wire"), and $0.32 \mathrm{~ns}$ (fully wetted).

through the simulation box. Under this potential, conduction breaks were noted in the simulation from dry nonconductive to water-and ion-conducting pores, akin to the higher voltage breaks seen with the constant electric field. This resulted in rapid wetting of the nanopore (Figure 2), which was maintained throughout the $50 \mathrm{~ns}$ duration of the simulation.

Electrowetting Precedes Ion Permeation. We investigated in more detail the onset of electrowetting in the $\Delta V=$ $1.2 \mathrm{~V} \mathrm{CE}$ simulation of the $\beta 14 \mathrm{~L} 3$ nanopore by tracking the individual trajectories of water molecules and of ions within the transbilayer pore (Figure 3). It can be seen that the initial wetting event is due to water entry into the hydrophobic central region of the pore (the same is also seen in the constant field simulations; data not shown). Following wetting of the pore (within less than a nanosecond of the start of the simulation with an applied $\Delta V$; Figure 3) ions enter the pore and multiple ion conduction events occur over the course of the simulation. We wished to test the robustness of this result to the force field and water model used. We therefore repeated this simulation (which used the GROMOS $43 \mathrm{a} 1^{44,45}$ force field and the threesite SPC water model), ${ }^{46}$ instead using the OPLS UA force field ${ }^{47}$ and the four-site $\mathrm{TIP}_{4} \mathrm{P}^{48}$ water model. The results obtained with OPLS UA and TIP4P were very similar to those for the GROMOS 43a1 and SPC simulations, namely, that wetting of the $\beta 14 \mathrm{~L} 3$ nanopore was observed within the first $0.5 \mathrm{~ns}$ of the simulation (see SI Figure S3). It therefore seems the key observation is robust to changes in the force field parameters and the water model employed. Our simulation have been performed with a bulk ionic concentration of $1 \mathrm{M}$; this is quite typical for experimental studies of nanopore gating (see, e.g., ref 7). However, it has recently been shown ${ }^{31}$ for a hydrophobic gate in a biological ion channel that the free energy landscape for water permeation (and in particular the barrier height to permeation presented by a hydrophobic gate) does not change substantially when the bulk ionic concentration is changed from $1.0 \mathrm{M}$ to $0.15 \mathrm{M}$.

Water entry in response to an applied voltage into the hydrophobic regions of simplified models of pores ${ }^{23,25}$ and carbon nanotubes ${ }^{27}$ has been studied previously, although most such studies employed the constant field method rather than a potential difference generated by asymmetric ion distributions. We therefore wished to verify that a similar mechanism of electrowetting occurred in our model protein nanopores.

Water dipole alignment within the electrowetted $\beta 14 \mathrm{~L} 3$ nanopore was compared with that within a $\beta 14 \mathrm{~L} 1$ nanopore, which wets in the absence of an applied voltage (Figure 4). The $\beta 14 \mathrm{~L} 1$ pore has just a single ring of leucine residues and therefore is fully wetted in the absence of an applied voltage difference across the membrane, as described in a previous paper $^{43}$ (in which $\beta 14 \mathrm{~L} 1$ is referred to as $N=14$, STNLNTS). It can be seen that the water dipoles are highly aligned in the center of the $\beta 14 \mathrm{~L} 3$ nanopore by the transbilayer electric field, in agreement with a number of other pore studies. ${ }^{25,27}$ In contrast, in the absence of a transbilayer voltage difference in the $\beta 14 \mathrm{~L} 1$ nanopore, water molecules only partially oriented in the region of the lipid headgroup dipoles. Locally oriented water molecules have been seen in a number of simulations of biological pores, e.g., for bacterial porins, ${ }^{49}$ and within the aquaporins, ${ }^{50,51}$ but not generally in the context of electrowetting (but see ref 29). Thus, the transbilayer voltage induces the formation of a locally oriented water wire in the center of the nanopore (see the inset in Figure 4), which in turn leads to water and ion permeation through the "open" pore.

Reversibility of Electrowetting. A decrease in voltage below the threshold value of wetting has been shown to expel water from hydrophobic solid-state nanopores ${ }^{13}$ and in simulations of electrowetted simplified nanopores ${ }^{38}$ and carbon nanotubes. ${ }^{27}$ To explore this for the electrowetted $\beta 14 \mathrm{~L} 3$ nanopore, the final configuration of the $\Delta V=1.2 \mathrm{~V}$ simulation system was taken, and the CE simulation protocol "switched off" such that as ions moved through the open pore, the transbilayer voltage difference dissipated. After a number of ion conduction events (Figure 5) both of the pores in the simulation system dewetted, preventing further permeation. Measuring the electric field along the bilayer normal after both pores had closed revealed that the transbilayer voltage had dropped to $\Delta V=0.2 \mathrm{~V}$. Of course, once the pores had closed, this could not return to $\Delta V=0 \mathrm{~V}$, as ions were unable to cross the membranes. Detailed inspection of the ion and water trajectories suggested that once the pore has dewetted, no further transient permeation events occur over the duration (50 ns) of the simulation. This is consistent with earlier studies, which have indicated that while dewetting of hydrophobic nanopores may be thermodynamically favorable, it is predicted to be feasible kinetically only in nanopores with diameters of less than a few nanometers. ${ }^{52}$

Voltage Dependence of Electrowetting. Simulations were conducted with different transbilayer voltages for the $\beta 14 \mathrm{~L} 3$ nanopore, using both the CE (Figure 6A) and constant field (SI Figure S2B) methods. Both methods revealed a clear relationship between the transbilayer voltage difference and the wetting of the nanopore with a threshold voltage (measured as the voltage difference yielding half-maximal wetting of the nanopore) of $\Delta V_{0.5}=1.0 \mathrm{~V}$. (This is the estimate from the CE simulations; the constant field simulations yielded a slightly 


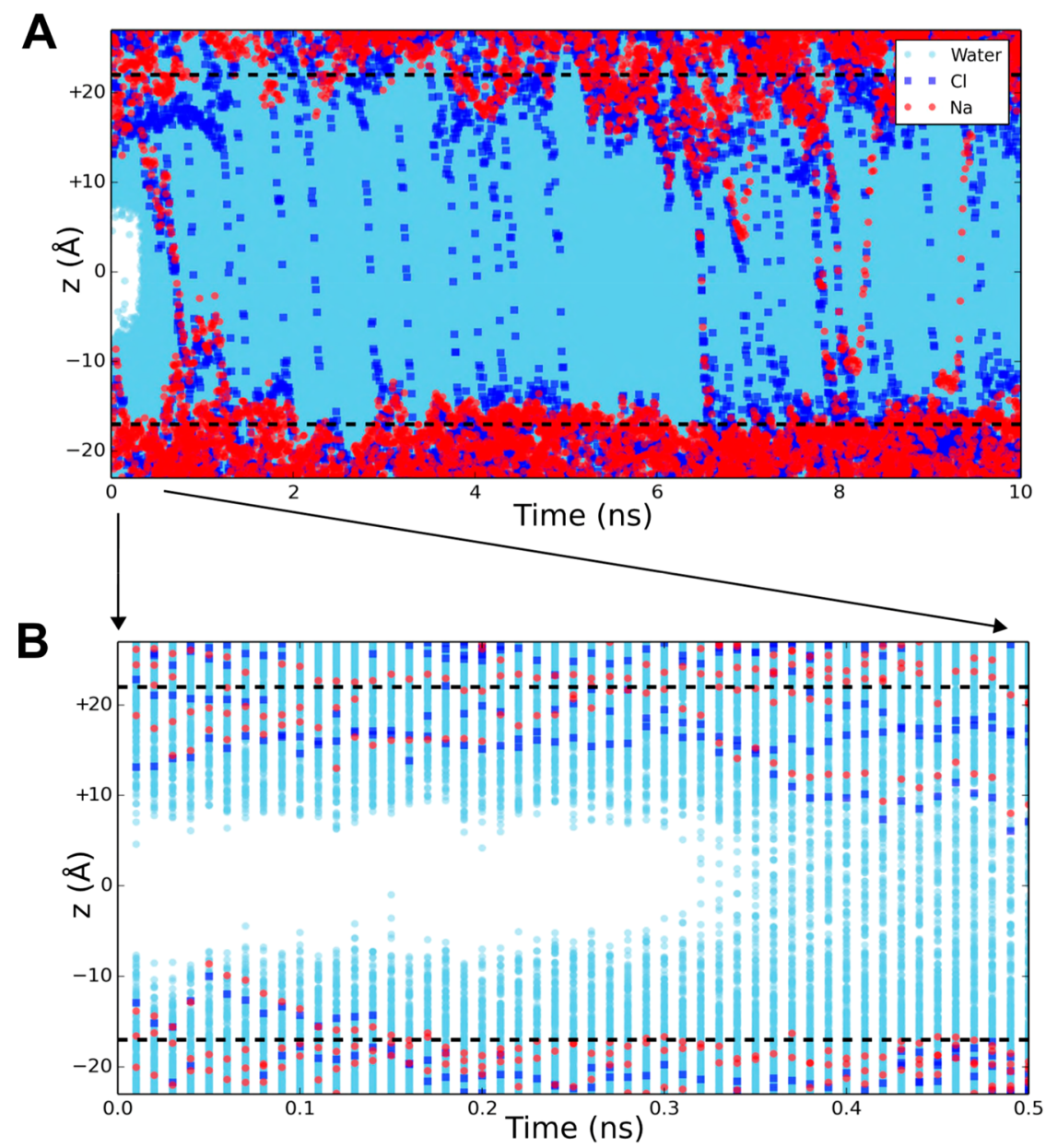

Figure 3. Trajectories for water molecules and ions entering the $\beta 14 \mathrm{~L} 3$ pore at $\Delta V=1.2 \mathrm{~V}$. (A) Plot of the $z$ position of a water molecule (pale blue) or ion ( red $=\mathrm{Na}^{+}$; blue $=\mathrm{Cl}^{-}$) as a function of simulation time. The dashed horizontal lines indicate the average $z$ position of the phosphate atoms of the lipid headgroups. The white region until $0.3 \mathrm{~ns}$ indicates the initial dewetted region within the central hydrophobic barrier formed by three rings of leucine residues. (B) Zoomed-in view of the first $0.5 \mathrm{~ns}$ of the trajectory shown in A.

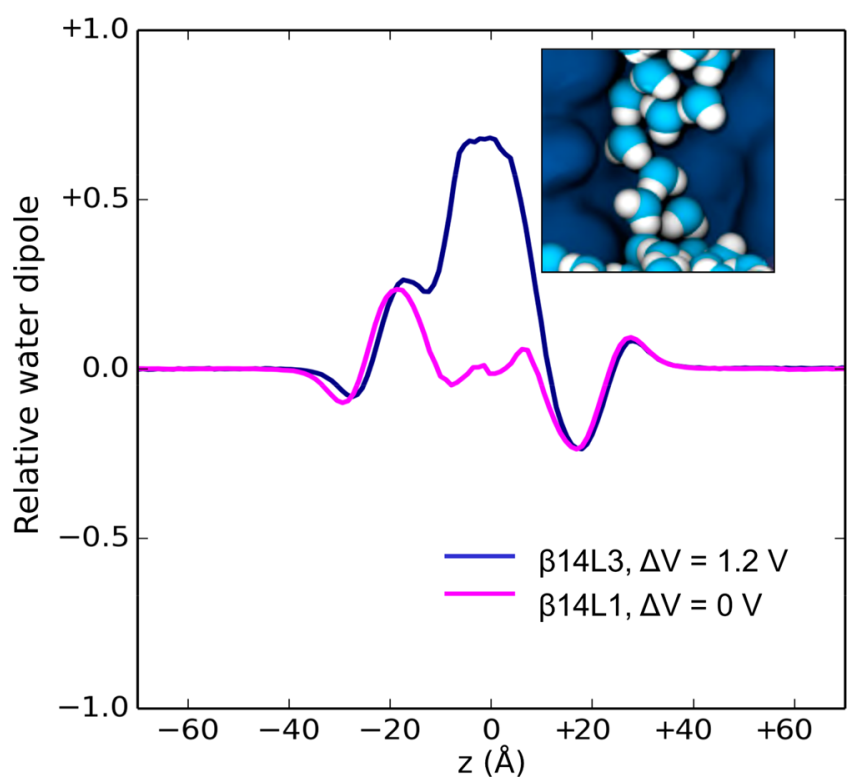

Figure 4. Water molecule dipolar alignment within the $\beta 14 \mathrm{~L} 3$ pore at $\Delta V=1.2 \mathrm{~V}$ (blue) and for a control pore, $\beta 14 \mathrm{~L} 1$ (pink), which has just a single ring of leucine residues and which is fully wetted in the absence of an applied voltage difference across the membrane. lower value of $\Delta V_{0.5}=0.8 \mathrm{~V}$. In part the discrepancy is related to the difficulty of exactly defining the transbilayer voltage drop in the constant field simulations; see SI Figure S1.) At the lower voltages $(\Delta V c a .0 .8 \mathrm{~V})$ the onset of electrowetting seems to take longer.

The number of ion permeation events observed as a function of the transbilayer voltage may be used to estimate the conductance of the $\beta 14 \mathrm{~L} 3$ nanopore, yielding an estimate of $460( \pm 220)$ pS (in $1 \mathrm{M} \mathrm{NaCl}$; see SI Table S5 for the individual conductance values estimated at different transbilayer voltages). This may be compared with the experimental value of $1200 \mathrm{pS}$ for the OmpG pore (in $1 \mathrm{M} \mathrm{KCl}$ ), which is also formed by a 14-stranded $\beta$-barrel, albeit with a somewhat higher pore radius due to the absence of a hydrophobic gate. Comparison of the number of permeation events for sodium and chloride ions indicates that the $\beta 14 \mathrm{~L} 3$ nanopore is anion selective, even though there are no charged side chains present in the lining of the pore. This may be due to the penetration of sodium ions into the headgroup region of the bilayer (which has been observed in other simulations ${ }^{53}$ ), which in turn results in the interior of the bilayer having a potential of $c a .+0.8 \mathrm{~V}$ relative to the adjacent aqueous solution irrespective of the transbilayer voltage (see SI Figure S1A).

Electrowetting Does Not Occur for the $\beta 16 \mathrm{~L} 5$ Nanopore. The CE method was also used to explore possible 


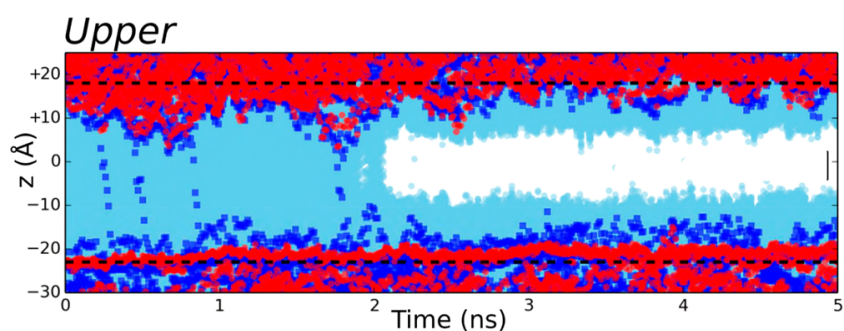

Lower

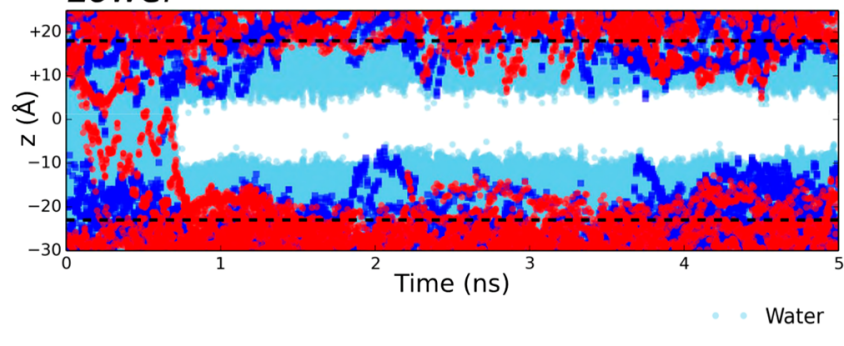

Figure 5. Trajectories for water molecules and ions from a simulation of the $\beta 14 \mathrm{~L} 3$ pore, which starts with $\Delta V=1.2 \mathrm{~V}$, but in which the voltage difference across the membrane is allowed to dissipate over the first few ns of the simulation. The upper and lower panels refer to the upper and lower bilayers as shown in Figure 1B. The color code is as in Figure 3.

electrowetting of the $\beta 16 \mathrm{~L} 5$ nanopore, which although wider (radius ca. $6.5 \AA$ ) has a more extensive hydrophobic gate. In this case, application of transbilayer voltages up to $\Delta V>2.5 \mathrm{~V}$ did not result in electrowetting of the central pore (Figure 6B). Although electrowetting did not occur at the higher voltages, a degree of voltage-induced pore deformation occurred, and also electroporation of the lipid bilayer took place, as has been seen in a number of simulation studies (e.g., refs 54 and 55). For reference, for the $\beta 14 \mathrm{~L} 3$ pore at the highest voltage differences studied $(\Delta V=1.2 \mathrm{~V})$, although occasional voltage-induced distortion of the nanopore leading to its "collapse" of the nanopore (SI Figure S4) occurred (as has been seen in some porins ${ }^{56}$ ), electroporation of the lipid bilayer was not seen.

\section{CONCLUSIONS}

We have demonstrated, using realistic simulation of transbilayer voltages via computational electrophysiology, that hydrophobic gates in model $\beta$-barrel nanopores can be functionally opened by electrowetting at voltages that do not electroporate lipid bilayers (Figure 7). We show that electrowetting occurs due to voltage-induced alignment of water dipoles within the hydrophobic gate region of the nanopores and that water entry occurs prior to entry of ions into the opened nanopore. If the ionic concentration gradient generating the transbilayer potential is allowed to dissipate, the electrowetting is reversed, water is expelled, and the nanopore reverts to a dewettted, functionally closed state. The dependence of functional "openness", measured as the time-averaged degree of electrowetting, as a function of $\Delta V$ is sigmoidal. The electrowetting can be "tuned" via the radius of the pore and the extent of the hydrophobic gate. Thus, for a more extensive hydrophobic gate within a given pore electrowetting does not occur before voltages that lead to the onset of electroporation of a lipid bilayer. The functionally open channel formed by electrowetting of a "featureless" $\beta$-barrel nanopore is anionic selective due to the transmembrane dipole potential caused by binding

\section{A}
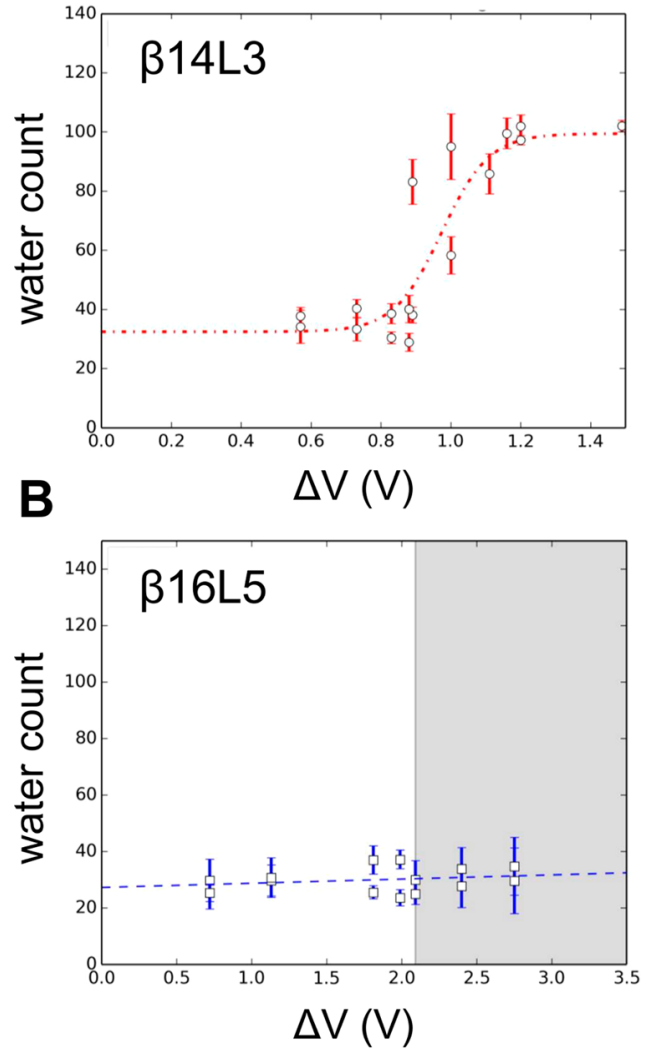

Figure 6. (A) Electrowetting of the $\beta 14 \mathrm{~L} 3$ pore as a function of transmembrane voltage. Electrowetting is monitored via the number of waters within the leucine region of the pore. The data are fitted as a sigmoid function with $\Delta V_{0.5}=1.0 \mathrm{~V}$. Error bars are calculated from standard error of the mean from water count within the pore. Two points for a given value of $\Delta V$ correspond to the "upper" and "lower" pore of the two bilayer system. A single point indicates only one stable conductive pore was present in the simulation (see text for details). (B) Computational simulations of the $\beta 16 \mathrm{~L} 5$ pore as a function of voltage. The gray region indicates voltages that resulted in breakdown of the DPPC bilayer.

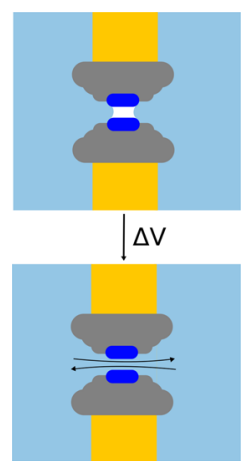

Figure 7. Schematic representation of electrowetting of a hydrophobic barrier to yield a voltage-gated nanopore. Pale blue = electrolyte solution; yellow = lipid bilayer membrane; gray = nanopore; deep blue = hydrophobic gate; white $=$ dewetted region within the hydrophobic gate.

of $\mathrm{Na}^{+}$ions to the headgroup regions of the surrounding lipid molecules.

There are a number of forward-looking implications of this work. It is clear that hydrophobic barriers can provide voltage- 
dependent gates in designed biomimetic nanopores and that these gates can be "tuned" to provide a specified threshold voltage for pore gating. Furthermore, one might anticipate that the onset of electrowetting (observed on a subnanosecond time scale) of a nanopore would occur more quickly than, for example, a conformational change linked to gating of a protein nanopore or ion channel on a submillisecond time scale. However, it is clear that further studies are required to characterize the interplay between pores, bilayer lipids, and ion selectivity that is observed even for relatively simple nanopore systems. This work has extended our understanding of hydrophobic gating in a number of synthetic and biological nanopores and provides a framework for the design of functional nanopores with tailored gating functionality.

\section{METHODS}

Models and Simulation Systems. The $\beta 14 \mathrm{~L} 3$ and $\beta 16 \mathrm{~L} 5$ model pores were modeled based on idealized $\beta$-barrel pores ${ }^{57}$ using MODELLER $^{58} 9 \mathrm{v} 9$ as described in a previous paper. ${ }^{43}$ Thus, the $\beta 14 \mathrm{~L} 3$ model in the current study corresponds to the $N=14, \mathrm{HG}$, STLLLTS model of the previous study. A single model pore was embedded in a lipid bilayer containing 400 DPPC molecules and solvated with $\sim 44000$ water molecules along with $\mathrm{Na}^{+}$and $\mathrm{Cl}^{-}$ions to a final ionic strength of $1 \mathrm{M}$. This system was duplicated for the $\mathrm{CE}$ simulations (see SI Figure S1B) to yield two membrane systems with two copies of the protein, $\sim 800$ DPPC lipids and $\sim 88000$ waters.

Computational Electrophysiology. The double-membrane system was set up as shown in Figure 1B and SI Figure S1B. This was used to generate an initial ionic imbalance, which was maintained by an ion replacement protocol as described by ref 42 . The potential difference across the membrane was determined from the electrostatic potential along the $z$ axis (i.e., normal to the bilayers; Figure 2), which in turn was obtained by integrating twice the charge density along $z$.

MD Simulations. Simulations were performed using GROMACS (www.gromacs.org) version 4.6.5 $5^{59}$ with the GROMOS96 $43 \mathrm{al}$ force field. ${ }^{44,45}$ Long-range electrostatic interactions were treated with the Particle Mesh Ewald method ${ }^{60,61}$ with a short-range cutoff of $1 \mathrm{~nm}$ and a Fourier spacing of $0.12 \mathrm{~nm}$. The SPC model was used for water. ${ }^{46,62}$ Simulations were performed in the NPT ensemble with the temperature being maintained at $310 \mathrm{~K}$ with a v-rescale thermostat ${ }^{63}$ and a coupling constant of $\tau_{\mathrm{T}}=0.1$ ps. Pressure was maintained semiisotropically using the Parrinello-Rahman algorithm ${ }^{64}$ at 1 bar coupled at $\tau_{\mathrm{p}}=1$ ps. The time step for integration was $2 \mathrm{fs}$ with bonds constrained using the LINCS algorithm. ${ }^{65}$ Simulations were run for $100 \mathrm{~ns}$ using constant electric field and $50 \mathrm{~ns}$ for CE. Simulation results were analyzed using GROMACS, MDAnalysis, ${ }^{66}$ and locally written code. Molecular visualization used VMD. ${ }^{67}$ Pore radius profiles were estimated using HOLE. ${ }^{68}$

\section{ASSOCIATED CONTENT}

\section{S Supporting Information}

The Supporting Information is available free of charge on the ACS Publications website at DOI: 10.1021/acsnano.6b07865.

$$
\text { Additional data analysis (PDF) }
$$

\section{AUTHOR INFORMATION}

\section{Corresponding Author}

*E-mail: mark.sansom@bioch.ox.ac.uk.

\section{ORCID}

Mark S. P. Sansom: 0000-0001-6360-7959

\section{Present Addresses}

${ }^{\S}$ Department of Physics, Kings College London, Strand, London WC2R 2LS, U.K.

${ }^{\perp}$ Center for Quantitative Biology \& Peking-Tsinghua Center for Life Sciences, Peking University, Beijing, 100871, China.

\section{Notes}

The authors declare no competing financial interest.

\section{ACKNOWLEDGMENTS}

J.L.T. was supported by an iCASE studentship from BBSRC (grant number BB/I015728/1) and Oxford Nanopore Technologies. M.S.P.S. acknowledges support from the Leverhulme Trust (grant number RPG-2013-393). C.S. was supported by a Marie Curie Intra European Fellowship within the 7th European Community Framework Programme (grant number 327051). Our thanks to Vishal Maingi for his comments on the manuscript.

\section{REFERENCES}

(1) Hou, X.; Jiang, L. Learning from Nature: Building Bio-Inspired Smart Nanochannels. ACS Nano 2009, 3, 3339-3342.

(2) Gu, L. Q.; Braha, O.; Conlan, S.; Cheley, S.; Bayley, H. Stochastic Sensing of Organic Analytes by a Pore-Forming Protein Containing a Molecular Adapter. Nature 1999, 398, 686-690.

(3) Bayley, H.; Cremer, P. S. Stochastic Sensors Inspired by Biology. Nature 2001, 413, 226-230.

(4) Corry, B. Designing Carbon Nanotube Membranes for Efficient Water Desalination. J. Phys. Chem. B 2008, 112, 1427-1434.

(5) Howorka, S.; Cheley, S.; Bayley, H. Sequence-Specific Detection of Individual DNA Strands Using Engineered Nanopores. Nat. Biotechnol. 2001, 19, 636-639.

(6) Manrao, E. A.; Derrington, I. M.; Laszlo, A. H.; Langford, K. W.; Hopper, M. K.; Gillgren, N.; Pavlenok, M.; Niederweis, M.; Gundlach, J. H. Reading DNA at Single-Nucleotide Resolution with a Mutant Mspa Nanopore and Phi29 DNA Polymerase. Nat. Biotechnol. 2012, 30, 349-U174.

(7) Chen, M.; Khalid, S.; Sansom, M. S. P.; Bayley, H. Outer Membrane Protein G: Engineering a Quiet Pore for Biosensing. Proc. Natl. Acad. Sci. U. S. A. 2008, 105, 6272-6277.

(8) Ghadiri, M. R.; Granja, J. R.; Buehler, L. K. Artificial Transmembrane Ion Channels from Self-Assembling Peptide Nanotubes. Nature 1994, 369, 301-304.

(9) Engels, M.; Bashford, D.; Ghadiri, M. R. Structure and Dynamics of Self-Assembling Peptide Nanotubes and the Channel-Mediated Water Organization and Self-Diffusion. A Molecular Dynamics Study. J. Am. Chem. Soc. 1995, 117, 9151-9158.

(10) Burns, J. R.; Stulz, E.; Howorka, S. Self-Assembled DNA Nanopores That Span Lipid Bilayers. Nano Lett. 2013, 13, 2351-2356.

(11) Göpfrich, K.; Li, C. Y.; Ricci, M.; Bhamidimarri, S. P.; Yoo, J.; Gyenes, B.; Ohmann, A.; Winterhalter, M.; Aksimentiev, A.; Keyser, U. F. Large-Conductance Transmembrane Porin Made from DNA Origami. ACS Nano 2016, 10, 8207-8214.

(12) Geng, J.; Kim, K.; Zhang, J. F.; Escalada, A.; Tunuguntla, R.; Comolli, L. R.; Allen, F. I.; Shnyrova, A. V.; Cho, K. R.; Munoz, D.; Wang, Y. M.; Grigoropoulos, C. P.; Ajo-Franklin, C. M.; Frolov, V. A.; Noy, A. Stochastic Transport through Carbon Nanotubes in Lipid Bilayers and Live Cell Membranes. Nature 2014, 514, 612-615.

(13) Powell, M. R.; Cleary, L.; Davenport, M.; Shea, K. J.; Siwy, Z. S. Electric-Field-Induced Wetting and Dewetting in Single Hydrophobic Nanopores. Nat. Nanotechnol. 2011, 6, 798-802.

(14) Beckstein, O.; Biggin, P. C.; Sansom, M. S. P. A Hydrophobic Gating Mechanism for Nanopores. J. Phys. Chem. B 2001, 105, 1290212905 .

(15) Beckstein, O.; Sansom, M. S. P. Liquid-Vapor Oscillations of Water in Hydrophobic Nanopores. Proc. Natl. Acad. Sci. U. S. A. 2003, 100, 7063-7068.

(16) Beckstein, O.; Sansom, M. S. P. A Hydrophobic Gate in an Ion Channel: The Closed State of the Nicotinic Acetylcholine Receptor. Phys. Biol. 2006, 3, 147-159.

(17) Aryal, P.; Abd-Wahab, F.; Sansom, M. S. P.; Tucker, S. J. A Hydrophobic Barrier Deep within the Inner Pore of the Twik-1 K2p Potassium Channel. Nat. Commun. 2014, 5, 4377. 
(18) Anishkin, A.; Sukharev, S. Water Dynamics and Dewetting Transitions in the Small Mechanosensitive Channel Mscs. Biophys. J. 2004, 86, 2883-2895.

(19) Anishkin, A.; Akitake, B.; Kamaraju, K.; Chiang, C. S.; Sukharev, S. Hydration Properties of Mechanosensitive Channel Pores Define the Energetics of Gating. J. Phys.: Condens. Matter 2010, 22, 454120.

(20) Beckstein, O.; Sansom, M. S. P. The Influence of Geometry, Surface Character and Flexibility on the Permeation of Ions and Water through Biological Pores. Phys. Biol. 2004, 1, 42-52.

(21) Beckstein, O.; Tai, K.; Sansom, M. S. P. Not Ions Alone: Barriers to Ion Permeation in Nanopores and Channels. J. Am. Chem. Soc. 2004, 126, 14694-14695.

(22) Allen, R.; Hansen, J. P.; Melchionna, S. Molecular Dynamics Investigation of Water Permeation through Nanopores. J. Chem. Phys. 2003, 119, 3905-3919.

(23) Dzubiella, J.; Allen, R. J.; Hansen, J. P. Electric Field-Controlled Water Permeation Coupled to Ion Transport through a Nanopore. J. Chem. Phys. 2004, 120, 5001-5004.

(24) Piasecki, J.; Allen, R. J.; Hansen, J. P. Kinetic Models of Ion Transport through a Nanopore. Phys. Rev. E 2004, 70, in press DOI: 10.1103/PhysRevE.70.021105.

(25) Dzubiella, J.; Hansen, J. P. Electric-Field-Controlled Water and Ion Permeation of a Hydrophobic Nanopore. J. Chem. Phys. 2005, $122.23470610 .1063 / 1.1927514$

(26) Hummer, G.; Rasaiah, J. C.; Noworyta, J. P. Water Conduction through the Hydrophobic Channel of a Carbon Nanotube. Nature 2001, 414, 188-190.

(27) Vaitheeswaran, S.; Rasaiah, J. C.; Hummer, G. Electric Field and Temperature Effects on Water in the Narrow Nonpolar Pores of Carbon Nanotubes. J. Chem. Phys. 2004, 121, 7955-7965.

(28) Rasaiah, J. C.; Garde, S.; Hummer, G. Water in Nonpolar Confinement: From Nanotubes to Proteins and Beyond. In Annual Reviews in Physical Chemistry; Annual Reviews: Palo Alto, 2008; pp 713-740.

(29) Spronk, S. A.; Elmore, D. E.; Dougherty, D. A. VoltageDependent Hydration and Conduction Properties of the Hydrophobic Pore of the Mechanosensitive Channel of Small Conductance. Biophys. J. 2006, 90, 3555-3569.

(30) Zhu, F. Q.; Hummer, G. Drying Transition in the Hydrophobic Gate of the Glic Channel Blocks Ion Conduction. Biophys. J. 2012, 103, 219-227.

(31) Trick, J. L.; Chelvaniththilan, S.; Klesse, G.; Aryal, P.; Wallace, E. J.; Tucker, S. J.; Sansom, M. S. P. Functional Annotation of Ion Channel Structures by Molecular Simulation. Structure 2016, 24, 2207-2216.

(32) Choudhuri, J. R.; Vanzo, D.; Madden, P. A.; Salanne, M.; Bratko, D.; Luzar, A. Dynamic Response in Nanoelectrowetting on a Dielectric. ACS Nano 2016, 10, 8536-8544.

(33) Bratko, D.; Daub, C. D.; Leung, K.; Luzar, A. Effect of Field Direction on Electrowetting in a Nanopore. J. Am. Chem. Soc. 2007, 129, 2504-2510.

(34) Daub, C. D.; Bratko, D.; Leung, K.; Luzar, A. Electrowetting at the Nanoscale. J. Phys. Chem. C 2007, 111, 505-509.

(35) Winarto; Takaiwa, D.; Yamamoto, E.; Yasuoka, K. Structures of Water Molecules in Carbon Nanotubes under Electric Fields. J. Chem. Phys. 2015, 142.12470110.1063/1.4914462

(36) Smirnov, S. N.; Vlassiouk, I. V.; Lavrik, N. V. Voltage-Gated Hydrophobic Nanopores. ACS Nano 2011, 5, 7453-7461.

(37) Aryal, P.; Sansom, M. S. P.; Tucker, S. J. Hydrophobic Gating in Ion Channels. J. Mol. Biol. 2014, 427, 121-130.

(38) Vanzo, D.; Bratko, D.; Luzar, A. Dynamic Control of Nanopore Wetting in Water and Saline Solutions under an Electric Field. J. Phys. Chem. B 2015, 119, 8890-8899.

(39) Gumbart, J.; Khalili-Araghi, F.; Sotomayor, M.; Roux, B. Constant Electric Field Simulations of the Membrane Potential Illustrated with Simple Systems. Biochim. Biophys. Acta, Biomembr. 2012, 1818, 294-302.
(40) Sachs, J. N.; Crozier, P. S.; Woolf, T. B. Atomistic Simulations of Biologically Realistic Transmembrane Potential Gradients. J. Chem. Phys. 2004, 121, 10847-10851.

(41) Melcr, J.; Bonhenry, D.; Timr, S.; Jungwirth, P. Transmembrane Potential Modeling: Comparison between Methods of Constant Electric Field and Ion Imbalance. J. Chem. Theory Comput. 2016, 12, 2418-2425.

(42) Kutzner, C.; Grubmueller, H.; de Groot, B. L.; Zachariae, U. Computational Electrophysiology: The Molecular Dynamics of Ion Channel Permeation and Selectivity in Atomistic Detail. Biophys. J. 2011, 101, 809-817.

(43) Trick, J. L.; Wallace, E. J.; Bayley, H.; Sansom, M. S. P. Designing a Hydrophobic Barrier within Biomimetic Nanopores. ACS Nano 2014, 8, 11268-11279.

(44) van Gunsteren, W. F.; Kruger, P.; Billeter, S. R.; Mark, A. E.; Eising, A. A.; Scott, W. R. P.; Huneberger, P. H.; Tironi, I. G. Biomolecular Simulation: The Gromos96 Manual and User Guide; Biomos \& Hochschulverlag AG an der ETH Zurich: Groningen and Zurich, 1996.

(45) Daura, X.; Mark, A. E.; van Gunsteren, W. F. Parametrization of Aliphatic Chn United Atoms of Gromos96 Force Field. J. Comput. Chem. 1998, 19, 535-547.

(46) Berendsen, H. J. C.; Postma, J. P. M.; van Gunsteren, W. F.; Hermans, J. Interaction Models for Water in Relation to Protein Hydration. In Intermolecular Forces; Pullman, B., Ed.; Reidel: Dordrecht, 1981; pp 331-342.

(47) Jorgensen, W. L.; Tirado-Rives, J. The Opls Potential Functions for Proteins - Energy Minimizations for Crystals of Cyclic-Peptides and Crambin. J. Am. Chem. Soc. 1988, 110, 1657-1666.

(48) Jorgensen, W. L.; Chandresekhar, J.; Madura, J. D.; Impey, R. W.; Klein, M. L. Comparison of Simple Potential Functions for Simulating Liquid Water. J. Chem. Phys. 1983, 79, 926-935.

(49) Acosta-Gutierrez, S.; Scorciapino, M. A.; Bodrenko, I.; Ceccarelli, M. Filtering with Electric Field: The Case of E. Coli Porins. J. Phys. Chem. Lett. 2015, 6, 1807-1812.

(50) de Groot, B. L.; Grubmuller, H. Water Permeation across Biological Membranes: Mechanism and Dynamics of Aquaporin-1 and Glpf. Science 2001, 294, 2353-2357.

(51) Tajkhorshid, E.; Nollert, P.; Jensen, M. O.; Miercke, L. J.; O'Connell, J.; Stroud, R. M.; Schulten, K. Control of the Selectivity of the Aquaporin Water Channel Family by Global Orientational Tuning. Science 2002, 296, 525-30.

(52) Leung, K.; Luzar, A.; Bratko, D. Dynamics of Capillary Drying in Water. Phys. Rev. Lett. 2003, 90, 065502.

(53) Pandit, S. A.; Bostick, D.; Berkowitz, M. L. Molecular Dynamics Simulation of a Dipalmitoylphosphatidylcholine Bilayer with Nacl. Biophys. J. 2003, 84, 3743-3750.

(54) Tarek, M. Membrane Electroporation: A Molecular Dynamics Simulation. Biophys. J. 2005, 88, 4045-4053.

(55) Böckmann, R. A.; de Groot, B. L.; Kakorin, S.; Neumann, E.; Grubmuller, H. Kinetics, Statistics, and Energetics of Lipid Membrane Electroporation Studied by Molecular Dynamics Simulations. Biophys. J. 2008, 95, 1837-1850.

(56) Zachariae, U.; Schneider, R; Briones, R.; Gattin, Z.; Demers, J. P.; Giller, K.; Maier, E.; Zweckstetter, M.; Griesinger, C.; Becker, S.; Benz, R.; de Groot, B. L.; Lange, A. Beta-Barrel Mobility Underlies Closure of the Voltage-Dependent Anion Channel. Structure 2012, 20, $1540-1549$

(57) Sansom, M. S. P.; Kerr, I. D. Transbilayer Pores Formed by BBarrels:- Molecular Modelling of Pore Structures and Properties. Biophys. J. 1995, 69, 1334-1343.

(58) Sali, A.; Blundell, T. L. Comparative Protein Modeling by Satisfaction of Spatial Restraints. J. Mol. Biol. 1993, 234, 779-815.

(59) Hess, B.; Kutzner, C.; van der Spoel, D.; Lindahl, E. Gromacs 4: Algorithms for Highly Efficient, Load-Balanced, and Scalable Molecular Simulation. J. Chem. Theory Comput. 2008, 4, 435-447.

(60) Darden, T.; York, D.; Pedersen, L. Particle Mesh Ewald - an N.Log(N) Method for Ewald Sums in Large Systems. J. Chem. Phys. 1993, 98, 10089-10092. 
(61) Essmann, U.; Perera, L.; Berkowitz, M. L.; Darden, T.; Lee, H.; Pedersen, L. G. A Smooth Particle Mesh Ewald Method. J. Chem. Phys. 1995, 103, 8577-8593.

(62) van der Spoel, D.; van Maaren, P. J.; Berendsen, H. J. C. A Systematic Study of Water Models for Molecular Simulation: Derivation of Water Models Optimized for Use with a Reaction Field. J. Chem. Phys. 1998, 108, 10220-10230.

(63) Bussi, G.; Donadio, D.; Parrinello, M. Canonical Sampling through Velocity Rescaling. J. Chem. Phys. 2007, 126, 014101.

(64) Parrinello, M.; Rahman, A. Polymorphic Transitions in SingleCrystals - a New Molecular-Dynamics Method. J. Appl. Phys. 1981, 52, $7182-7190$

(65) Hess, B.; Bekker, H.; Berendsen, H. J. C.; Fraaije, J. G. E. M. Lincs: A Linear Constraint Solver for Molecular Simulations. J. Comput. Chem. 1997, 18, 1463-1472.

(66) Michaud-Agrawal, N.; Denning, E. J.; Woolf, T. B.; Beckstein, O. Mdanalysis: A Toolkit for the Analysis of Molecular Dynamics Simulations. J. Comput. Chem. 2011, 32, 2319-2327.

(67) Humphrey, W.; Dalke, A.; Schulten, K. Vmd - Visual Molecular Dynamics. J. Mol. Graphics 1996, 14, 33-38.

(68) Smart, O. S.; Neduvelil, J. G.; Wang, X.; Wallace, B. A.; Sansom, M. S. P. Hole: A Program for the Analysis of the Pore Dimensions of Ion Channel Structural Models. J. Mol. Graphics 1996, 14, 354-360. 